\title{
Nunca hemos sido animales. El posthumanismo de Heidegger
}

\author{
We have never been Animals. \\ Heidegger's Posthumanism ${ }^{I}$
}

Steven Crowell ${ }^{2}$

Rice University, Estados Unidos

Recepción: 23 de julio del 2020

Evaluación: 31 de julio del 2020

Aceptación: 11 de agosto del 2020

1 Traducción de Carlos Rincón Zabala, Centro Internacional de Estudios sobre el Nihilismo Contemporáneo (CeNic).

2 Doctor por la Universidad de Yale y Magister en la Northen Illionis University. Actualmente es Profesor en la Rice University (USA).

Correo electrónico: crowell@rice.edu

Crowell, S. (2020). Nunca hemos sido animales. El posthumanismo de Heidegger.

Cuestiones de Filosofía, 6 (26), 165-193. 


\title{
Resumen
}

La pregunta por la relación entre el Dasein y el ser humano nunca fue adecuadamente caracterizada en Ser y tiempo. En la década siguiente a esta publicación, Heidegger examinó este problema cuando trata de elaborar una "metafísica" fundada fenomenológicamente. Un momento crucial en este intento se encuentra en su reflexión acerca del animal en su curso magistral de 1929/30, Conceptos fundamentales de la metafísica. Este escrito explora el tratamiento heideggeriano de la diferencia entre el animal (incluyendo al homo sapiens) y el "Dasein en nosotros". Esto es hecho en diálogo con un reciente ejemplo de lo que yo denomino "bio-posthumanismo", el punto de vista de que normativamente no existe una distinción significativa a considerar entre el ser humano y otros animales. Surge de esta manera una imagen del post-humanismo fenomenologico de Heidegger.

Palabras clave: vida, fenomenología, teoría de sistemas, posthumanismo, normatividad, metafísica.

\begin{abstract}
The question of the relation between Dasein and the human being was never adequately characterized in Being and Time. In the decade following that publication, Heidegger explored this problem as he tried to work out a phenomenologically based "metaphysics." A key moment in this attempt is found in his discussion of the animal in his 1929/30 lecture course, Fundamental Concepts of Metaphysics. This paper explores Heidegger's treatment of the difference between the animal (including homo sapiens) and the "Dasein in us." It does so in dialogue with a recent example of what I call "bio-posthumanism," the view that there is no normatively meaningful distinction to be drawn between human beings and other animals. A picture of Heidegger's phenomenological post-humanism emerges.
\end{abstract}

Keywords: life, phenomenology, systems theory, posthumanism, normativity, metaphysics. 
En su Carta sobre el humanismo (1946) Heidegger se mueve por una línea retórica entre rechazar y adoptar el "humanismo" como una etiqueta para su propio pensamiento. Por un lado, el término pertenece a una tradición (llamada aquí "metafísica") en la cual "el hombre es la medida de todas las cosas", tradición criticable por cierto para Heidegger. Por otro lado, a diferencia del humanismo tradicional (que no se percata aún "de la verdadera dignidad del hombre"), el "pensamiento del ser" no-metafísico de Heidegger pretende hacer justicia a la condición particular del hombre como "pastor del ser" (Heidegger, 1993, pp. 225, 233, 234).

El presente ensayo pretende desarrollar algunas de las razones e implicaciones de esta aporía retórica. Eso hace que el título "posthumanismo de Heidegger", más que "humanismo heideggeriano", tenga que ver con el camino que el ulterior pensamiento continental ha tomado. Recurriendo a los tratados conocidos de Foucault (1973) y Derrida (1982), esta reflexión rechaza el "subjetivismo" fenomenológico y tiende a ver a Heidegger simplemente como otro humanista. A pesar de sus intentos de romper con la fenomenología "empírico-trascendental" -según esta argumentación-, Heidegger refuerza el humanismo metafísico cuando, por ejemplo, resalta el "abismo" que nos separa de lo que hemos solido llamar "otros" animales, y elogia, en su lugar, nuestra proximidad a "la esencia de lo divino" (Heidegger, 1993, p. 230).

La distinción normativa a la que este tipo de diferencia abismal parece conllevar, es el objetivo de cierto posthumanismo que admite muchas diferencias entre especies de seres vivos, pero no le concede a ninguno de ellos el tipo de estatus normativo que permitiría hablar de un abismo. En ¿What is Posthumanism?, Cary Wolfe sostiene, por ejemplo, que cualquiera que sea el camino adecuado para una "bioética", "no tiene sentido proponer la distinción entre humanos y animales (no importa cómo esté establecida esta diferencia)" (2010, p. 98). Con respecto a lo primero, denominado por él los "dos tipos de finitud" - concretamente, la finitud de la carne, "vulnerabilidad física, la encarnación y, finalmente, la muerte"-, a los cuales nos referiremos, no hay distinción que pueda ser concluyente ${ }^{3}$. Este posthumanismo no es

3 El pasaje completo en el que se definen estas dos tesis debe ser citado aquí, ya que nos ocuparemos de la primera en el apartado 3 y de la segunda en el 4. Comentando el trabajo de Cora Diamond, Wolfe sostiene que "hay dos clases de finitud, dos tipos de pasividad y vulnerabilidad. El primer tipo (vulnerabilidad física, encarnación, y finalmente la muerte) es paradójicamente inaccesible, 
un "transhumanismo" que se modele a sí mismo en el concepto de cyborg y proclame tecnologías postéticas que encumbren al ser humano más allá de su contingente constitución biológica: una "evolución diseñada" que promete nuestro "triunfante desencarnamiento" (Wolfe, 2010, pp. xiii, xv). Este es más bien un tipo de bioposthumanismo que se mueve en la dirección opuesta a "las fantasías de la encarnación y la autonomía heredada del humanismo mismo" (Wolfe, 2010, p. xv). Para Wolfe, quien recurre a la deconstrucción y a la teoría de sistemas de segundo orden -a Derrida y a Luhmann-, el "espíritu" de la Ilustración implica un giro posthumanista, un rechazo de los "dogmas" y los "universales antropológicos" tomados prestados de "la religión, la ciencia y la política", las "represiones culturales y las fantasías, los protocolos filosóficos y las evasiones del humanismo como un fenómeno históricamente específico" (Wolfe, 2010, p. xvi). Esto requiere rechazar, en particular, el "tipo de subjetividad normativa" con la cual la filosofía (y con esto él quiere decir filosofía como tal, a diferencia de la "teoría"4) busca tratar asuntos, por ejemplo, en bioética. Esto significa "que la naturaleza del pensamiento mismo debe cambiar si pretende ser posthumanista" (Wolfe, 2010, p. xvi).

Los lectores de Heidegger encontrarán familiares tales indicaciones. Con escritos como "El final de la filosofía y la tarea del pensar" en mente, podemos ser dispensados por considerar que el pensamiento de Heidegger cuenta como "posthumanista" en el sentido de Wolfe 5 . Y, de hecho, como veremos, hay una considerable superposición entre la fenomenología heideggeriana y el bioposthumanismo, cuando éste aborda la pregunta acerca del animal. Aun así, el posthumanismo de Heidegger (si éste es eso) está excluido de

inapropiable, para nosotros, por la misma razón que la hace accesible -concretamente, un segundo tipo de "pasividad" o "no estar disponible"-, que es la finitud que experimentamos en nuestro sometimiento a un tecnicismo o mecanicismo radicalmente a-humano del lenguaje, un tecnicismo que tiene profundas consecuencias, por supuesto, para lo que apresuradamente también pensamos de 'nuestros' conceptos, que no son, por tanto -en un sentido relevante- 'nuestros' en lo absoluto" (Wolfe, 2010, p. 88).

4 La "teoría" es la respuesta a "la pregunta en el despertar del escepticismo", según lo analizado por Stanley Cavell: "lo que puede significar hacer filosofía después de la filosofía, en un cierto sentido, ¿se ha vuelto imposible?” (Wolfe, 2010, p. 70).

5 Por ejemplo, ya en el primer párrafo de este escrito Heidegger sostiene: "El título nombra el intento de una meditación que se queda en pregunta. Las preguntas son caminos para una respuesta. Esta consistiría -en el caso de que alguna vez se accediera a ella- en una transformación del pensar, no en un enunciado acerca de un contenido" (Heidegger, 1972, p. 55; 2000, p. 77; mi énfasis). 
este canon por lo que parecen ser, de alguna manera, fundamentos dudosos: concretamente que Heidegger rechaza la idea (central para el bioposthumanismo) de que la vida o la animalidad proveen de algo así como una piedra angular ético-teórica ${ }^{6}$. Si un transhumanismo del cyborg puede ser resumido con el eslogan "ya no somos más animales", el posthumanismo de Heidegger debe ser entendido como una suposición de que nunca hemos sido animales. Esta paradoja está ampliamente tratada en el curso de 1929/30 Los conceptos fundamentales de la metafísica. En este producto maduro de su década metafísica ${ }^{7}$, Heidegger defiende una forma de posthumanismo fenomenológico, que es tan consistente con la posición de Ser y tiempo como lo es con el pensamiento de Heidegger en Carta sobre el humanismo, para adoptar su maniobra retórica. Pero a lo largo de este curso Heidegger insiste en que la fenomenología solo puede llevarnos a un punto donde podamos ver qué es lo que está en juego; ella no puede dar lugar a la decisión que surge entre pensar que nosotros siempre hemos sido animales o que nunca lo hemos sido (Heidegger, 1995, pp. 355- 425).

\section{Fenomenología, vida y el problema del mundo}

Empecemos con el método que Heidegger emplea en el texto. Heidegger quiere conducir a sus estudiantes hacia un conocimiento del "mundo", y con este objetivo plantea una distinción entre entidades que son "sin mundo" (como la piedra), entidades que son "pobres de mundo" (y que él llama "animales") y entidades que son "configuradoras de mundo" (reciben aquí el nombre de "hombre" [Mensch]. Tal nombre es engañoso, ya que el argumento subsecuente señala que no es el ser humano, sino el Dasein en el ser humano el "configurador-de-mundo"). ¿Qué significa la "pobreza-de-mundo" de los animales? Heidegger recuerda a sus oyentes que responderá a esta pregunta, practicando la fenomenología: un "modo de ver y comprender que resulta ajeno y molesto al entendimiento común”, un "preguntar y comprender (...)

6 Para algunas comparaciones fenomenológicas confróntese: Weigelt (2002), Barbaras (2006), Lawlor (2006), Toadvine (2009) y Figal (2010). Ya en 1929 Heidegger advertía en contra de esta tentación siempre presente, criticando el intento de Max Scheler por desarrollar una "visión de mundo biológica" que interpretara "al ser humano" desde "la perspectiva de la vida" (Heidegger, 2007, p. 242).

7 Acerca de la designación "década metafísica", véase Crowell (2001, cap. 12), donde también se explica por qué Heidegger renuncia al enfoque metafísico, apenas asociable a sus convicciones fenomenológicas. 
anterior a toda argumentación", que fue "vuelto a despertar, y en su sentido más radical, a través de Husserl” (Heidegger, 1995, p. 232).

Heidegger acepta la esencia del animal con el fin de entender al ser humano (1995, pp. 169-173) y aplaude a la biología contemporánea por resistir a la reducción de las categorías "ontológico-regionales" de la física (1995, p. 188). Pero ¿qué clase de categorías regionales y ontológicas pertenecen a la vida? Aunque "no podemos separar la investigación positiva y metafísica" (Heidegger, 1995, p. 189), una metafísica de la vida no debe, sin embargo, abdicar de sus propios intereses por convertirse en una visión de mundo biológica (o zoológica). La fenomenología heideggeriana se acerca al asunto a través de la idea de "transposición", explicada en contraste con la "empatía" (1995, pp. 202-206). Si la empatía es entendida para abarcar cierta distancia entre mi consciencia y la de mi prójimo, depende ya en sí misma de la "transposición", es decir, de nuestro "poder de acompañar al otro ente aun siendo distintos de él" (1995, p. 203). Como Mitsein, mi ser en el mundo incluye ya siempre la transposición "en" otros que no son yo. ¿Se mantiene lo mismo para animales no humanos? El hecho de que nosotros hayamos sido transpuestos en otros seres humanos, junto con el hecho de que los seres humanos sean seres vivos, ¿significa que "la transponibilidad en otros animales, en lo vivo", pertenece a "la esencia del ser humano"? (1995, p. 209).

Sí y no. En la base de la "vida”, el “(...) animal (...) permite y exige esta transponibilidad del hombre en él, (...) sin embargo a su vez niega al hombre la posibilidad de acompañarlo (Heidegger, 2007, p. 261). Este rechazo es la "pobreza" de mundo del animal, su "tener y, no obstante, no tener" (Heidegger, 1995, p. 210; 2007, p. 280). Si como Heidegger admite "esta pobreza solo puede ser captada si sabemos previamente qué es el mundo" -por consiguiente, de una manera que está tácitamente orientada hacia el ser humano-, esto no debe impedir cierto tipo de intento fenomenológico por elucidar positivamente "la esencia de la pobreza de mundo", al clarificar "la animalidad misma" (1995, p. 211; 2007, p. 262). Al explorar lo que significa decir que el animal invita y rechaza nuestra transponibilidad, la fenomenología heideggeriana plantea la pregunta acerca de si las categorías de la vida son adecuadas para entender el mundo ${ }^{8}$.

8 Para un tratamiento general de la ambigüedad del concepto de vida en la fenomenología trascendental y algunas de sus críticas, confróntese Crowell (2014). 
Heidegger se acerca al concepto de vida a través del concepto de organismo, y al concepto de organismo a través del concepto de órgano". Un órgano, que tiene la "capacidad" (Fähigkeit) para cumplir alguna función determinada, difiere de una herramienta, que está disponible para cumplir una función. Un bolígrafo que está "listo" para escribir no tiene una capacidad para ello, ya que para funcionar debe ser empleado de acuerdo a la "prescripción" (1995, p. 228; 2007, p. 280) que deriva de una totalidad respeccional (Bewandtnisganzheit) o "mundo" (1995, p. 215; 2007, p. 266). Un órgano, en contraste, no requiere de esta totalidad de implicaciones. Más bien, su función está prescrita -y así, su mismo ser como órgano está constituido- a través de su incorporación en un organismo: "El organismo tiene capacidades", mientras que el órgano mismo es "una posesión de una capacidad" (1995, pp. 221-222; 2007, p. 273). Heidegger ilustra esto con referencia a organismos unicelulares cuyos órganos son "temporales"; la misma "abertura" sirve primero como una boca, luego como estómago, luego como intestino y, finalmente, como ano.

Las bases para esta diferenciación de capacidades en un órgano residen en el ser del organismo gobernado por un "impulso" o instinto (Trieb), que "siempre se ha pro-puesto el posible ámbito de realización” (1995, p. 228; 2007, p. 281). El carácter prescriptivo del impulso del organismo es la esencia de éste y, en términos más generales, la esencia de la "vida" (1995, p. 235; 2007, p. 287). La "particularidad" de un organismo consiste en este modo de ser-una-capacidad de cierto tipo. Este punto es fundamental para la explicación heideggeriana de la vida. Antes de profundizar en ella será útil traducirla al lenguaje teórico-sistémico del bioposthumanismo, enfatizando de esta manera el carácter fenomenológico del posthumanismo de Heidegger. De acuerdo al bioposthumanismo, lo que Heidegger llama 'la capacidad del animal' se entiende mejor como un código recurrente que funciona para reducir la abrumadora complejidad del entorno animal. Esto, tal como Heidegger sostiene, cede el paso a un "mundo".

9 Los detalles de la discusión de Heidegger en torno a la vida del animal en el curso de 1929/30 son esclarecedores y fiablemente expuestos en McNeill (1999). Más adelante en este trabajo retornaré a algunos desacuerdos interpretativos con McNeill. 


\section{Propuesta bio-posthumanista del mundo de Cary Wolfe}

El bio-posthumanismo no inicia como una imposición directa de las categorías biológicas sobre la ontofilosofía, sino como una "forma-de-teoría" que prescinde de las presumibles aspiraciones fundamentalistas de la filosofía -tanto racionalistas como empiristas- a favor de una distinción entre "sistema" y "entorno". El mundo circundante no es un ámbito determinado y dado con antelación (por ejemplo, la naturaleza), sino que es, paradójicamente, el entorno de un sistema dado (Wolfe, 2010, p. xiv). Su "diferencia" con el sistema reside en su abrumadora complejidad, relativa a las condiciones que definen el sistema. Estas condiciones yacen en el código interno del sistema, en su manera de reducir esa complejidad a través de la "selectividad" (Wolfe, 2010, p. 14). Lo que hace que un sistema sea sistema es su autorreflexividad o recursividad -la manera en que sus resultados se convierten, uno por uno, en entradas que proveen las bases para nuevos resultados (Wolfe, 2010, p. 111). De esta manera, el sistema puede ser llamado "autopoiético" o autorreproductivo (Wolfe, 2010, p. xxiii) ${ }^{10}$. Además, el carácter recursivo del código implica que tal sistema produce una clase de apertura en las configuraciones, alguna vez nuevas, una "apertura a través de la clausura" (Wolfe, 2010, p. xxi). Ya que el entorno no es algo neutral que se da por hecho, sino que incluye los resultados del sistema mismo, "entra" al sistema en forma de una complejización interna que, a su vez, provee las bases para nuevas modalidades del sistema -para el aprendizaje, la evolución y el desarrollo.

La raíz de todo esto, sin embargo, es una paradoja que ha permitido acusaciones de solipsismo que recaen sobre la teoría de sistemas: la distinción entre sistema y entorno es una diferenciación que se hace solo dentro del sistema. Para usar uno de los ejemplos de Wolfe: el sistema legal es el que establece la distinción entre lo "legal" y su entorno (es decir, lo que es "lo otro de" lo legal). Su código se ramifica en una forma de sistema inmanente de complejidad (las varias subcategorías de lo legal), pero éste no puede determinar empíricamente la legitimidad de su distinción inicial codificada. Tampoco puede establecer internamente esa legitimidad, a través de una comprensión

\footnotetext{
${ }^{10}$ Como es usado en debates contemporáneos, el término "autopoiesis" remite al trabajo de Humberto Maturana, especialmente a su colaboración con Francisco Varela. Obsérvese, por ejemplo, Maturana y Varela (1996).
} 
autorreflexiva de su propio código ${ }^{11}$. Para Wolfe, esto es lo que hace de la teoría de sistemas una teoría en lugar de "filosofía". Esta teoría se concibe a sí misma como capaz de justificar la distinción inicial entre lo que está incluido y lo que está excluido, a través de algo parecido a una comprensión racional de sus propias condiciones de posibilidad (Wolfe, 2010, pp. xx-xxi). Este es también el punto en el que la teoría de sistemas une fuerzas con su "complemento", la deconstrucción, que recala en la misma "imposibilidad" de autoforjarse a través de su noción de "iterabilidad". Lo que es iterable (por ejemplo, el código recurrente) debe englobar "en un momento dado la necesidad de pensar tanto la regla como el evento", un evento que es heterogéneo, "y no simplemente opuesto al orden del ideal, de lo calculable, de lo puro, y así sucesivamente" (Wolfe, 2010, p. 12).

Necesitamos movernos desde estas consideraciones generales hacia algo que nos lleve más cerca del abordaje que hace Heidegger en torno al animal. El puente está provisto por el concepto de significado. ¿Cómo es entendido el significado desde la teoría de sistemas? Para empezar, se debe reconocer una "estricta separación de los sistemas psíquico y social como entidades discretas y autopoiéticas" (Wolfe, 2010, p. 19). El sistema psíquico (mente, consciencia individual) implica un código para reducir la complejidad del entorno, convirtiéndolo así en "información". La información difiere de una mera estimulación porque, al haber sido producida por el código, su condición de entorno ha sido "virtualizada" (Wolfe, 2010, p. 18) -esto es, ahora incluye "la presentación simultánea (...) de actualidad y posibilidad"; con esto se ha convertido en "significado" (Wolfe, 2010. p. 16). Luhmann escribe: "la totalidad de las referencias presentadas por cualquier objeto significativamente deseado, ofrece a la mano más que lo que pueda de hecho ser actualizado en cualquier momento" (Wolfe, 2010, p. 16). Pero el significado entorpece a menos de que permanezca abierto a la actualización de (algunas

\footnotetext{
11 "Lo que esto significa no es únicamente que todos los sistemas y todas las observaciones son autorreferenciales. También significa que, paradójicamente, la diferencia entre la autorreferencia y la referencia externa (o 'heterorreferencia') es en sí misma un producto de autorreferencia, en la misma manera en que el 'exterior' del entorno es siempre el exterior de un 'interior' específico. Este hecho, sin embargo, no puede ser observado por el sistema que, al mismo tiempo, quiere usar la distinción para llevar a cabo sus operaciones. Esa observación solo puede ser hecha por un observador de segundo orden, usando un código diferente (...), el cual, asimismo, debe permanecer 'ciego' ante la naturaleza paradójica de $s u$ distinción constitutiva, que solo puede ser revelada por otro observador, etc." (Wolfe, 2010, p. 113). En la sección final de este artículo discutiré acerca de una explicación diferente -heideggeriana- de lo que implica la autorreflexividad.
} 
de) esas referencias, en el curso de lo cual se convierte en "información", es decir, en algo nuevo. Lo crucial aquí es el hecho de que el significado no está limitado a la mente humana. No todo sistema produce significado. No obstante, Luhmann sostiene: "pero para aquellos que lo hacen, esta es la única posibilidad [para procesar la complejidad]. El significado se convierte para ellos en la forma del mundo y, consecuentemente, solapa la diferencia entre sistema y entorno" (Wolfe, 2010, p. 19).

No obstante, si el significado corresponde al sistema psíquico, también surge al interior del sistema social de acuerdo con los imperativos del código autopoiético propio de este último. Ese código está circunscrito por el concepto de comunicación. Dos sistemas psíquicos no se comunican el uno con el otro; por el contrario, hay un sistema (el social) en el cual el significado es una función de la manera en que ciertos eventos del entorno (digamos gestos y ruidos) están codificados de acuerdo a un esquema tripartito: información ("contenido"), expresión ("comportamiento") y entendimiento ("procesamiento": la diferencia entre información y expresión) (Wolfe, 2010, p. 22). Tal como el significado en el sistema psíquico, el significado en el sistema social implica la virtualidad, la demanda por la actualización de potencialidades implícitas en el evento comunicativo. Estos hechos le permiten a Luhmann tratar el significado como "la verdadera 'sustancia' de este nivel evolutivo emergente". El significado no está basado en el sistema psíquico ni en el social: "Es imposible encontrar una 'substancia de soporte' para el significado" (Wolfe, 2010, p. 20).

De esta manera, los sistemas psíquico y social son complejizaciones internas del sistema autopoiético del significado mismo, tal como el derecho fiscal y las leyes de inmigración son complejizaciones dentro del sistema autopoiético legal: "El significado se apoya en aquello que permite su propia reproducción autorreferencial. Y únicamente las formas de esta reproducción diferencian las estructuras psíquica y social" (Wolfe, 2010, p. 20). De hecho, "el significado permite a los sistemas psíquicos y sociales interpenetrarse, mientras protegen su autopoiesis" (Wolfe, 2010, p. 21). Esto se logra a través del lenguaje -algo que no es psíquico ni social, pero es "un fenómeno de segundo orden: un 'tipo de comunicación mediática simbólicamente generalizada"-, que es utilizado por esos sistemas al servicio de procesos de significado de primer orden (Wolfe, 2010, p. 22). Pero esto conlleva, a su vez, a una decisión. La diferencia, previamente discutida, entre información y significado dentro de un sistema, implica un cierto tipo de indecibilidad 
pragmáticamente determinada (Wolfe, 2010, p. 90)-concretamente, el hecho de que para que el significado sea información, más allá de la actualización de una expresión, debe llevar al horizonte de posibilidad que pertenece a la virtualidad del significado. Pero esto significa que "el sistema debe decidir", esto es, "procesar selectivamente la diferencia entre información y expresión”, es decir, entendimiento (Wolfe, 2010, p. 23).

En este punto encontramos una convergencia ulterior entre la teoría de sistemas y la deconstrucción, debido a que el lenguaje no está gobernado por ningún proceso social ni psíquico. Para Luhmann, la tecnología de la escritura, como opuesta a la lengua oral, "impone la clara distinción entre información y expresión". Pero la reflexión de Derrida acerca de "la estructura de huella de la escritura-comunicación" muestra que la distinción entre información y expresión no está "limitada solo al dominio de lo humano y lingüístico", sino que caracteriza lo "recursivo, las dinámicas iterables del significado" como tal (Wolfe, 2010, pp. 24-25). Toda comunicación, incluyendo el lenguaje, y así también todo pensamiento, está por consiguiente atada al sistema autopoiético del significado que ha emergido a través de la evolución biológica. Y es solamente por esta razón que el pensamiento, en términos de significado, debe convertirse en "posthumanista" en el mismo sentido que el bioposthumanismo concede a este término: una apelación a formas lingüísticas de comunicación desarrolladas específicamente en el homo sapiens, no es suficiente para poner un "corte" entre sistemas de significado-producido y significado-no-producido. Si es de este modo, entonces, no hay un fundamento en la teoría del significado para distinguir entre el "entorno" de un animal y el "mundo" de un ser humano: todo sería mundo.

A partir de aquí no hay sino un corto paso para la concreta aplicación del bioposthumanismo de la teoría de sistemas deconstructivista. Mientras que la teoría de sistemas no reconoce "naturaleza o algún otro hecho dado con anterioridad" (Wolfe, 2010, p. xxiv), la eliminación del "significado desde el dominio ontológicamente cerrado de la consciencia, de la razón, de la reflexión etc.", significa que nosotros (teóricos) podemos "recontextualizar" nuestras gastadas concepciones humanistas de la percepción, de la afectividad etc. "en términos de todo el sensorium de otros seres vivos y de sus propias maneras autopoiéticas de 'traer a presencia un mundo' -maneras que, puesto que nosotros mismos somos animales humanos, son parte de la historia evolutiva y psicológica, y del repertorio comportamental del mismo ser humano-" (Wolfe, 2010, p. xxv). Esta suposición -de que no somos soberanos 
dentro del "dominio ontológicamente cerrado de la consciencia, de la razón, de la reflexión etc.", y entonces el "mundo" solo podría ser entendido como un código de animal autopoiético-, es precisamente lo que el posthumanismo fenomenológico de Heidegger pone en cuestión. Puesto que por ahora debe estar claro que la teoría de sistemas y la deconstrucción pueden converger únicamente en que ellas atribuyen a la filosofía los pecados de un cierto tipo de fundacionalismo cartesiano-kantiano (individualismo) o hegeliano (social), este proyecto es, entonces, fácil de "descentrar", ya que estas dos opciones se neutralizan una con la otra. Pero ¿son ellas las únicas opciones disponibles para comprender filosóficamente el concepto de significado?

El obstáculo con el que tropiezan tanto Derrida como Luhmann es el cuidado (Sorge) fenomenológico de Heidegger, que Wolfe asimila al "idealismo" de Descartes (2010, p. 41). Hacer esto le permite a Wolfe descartar el posthumanismo de Heidegger e introducir el concepto ontológico de "sujeto de una vida" del bioposthumanismo (2010, p. 66). Pero mientras que Heidegger estaría de acuerdo con que el significado no está anclado al sistema psíquico ni al sistema social como la teoría de sistemas los concibe, y mientras que Heidegger también estaría de acuerdo en que el significado es "virtual" -es decir, lo parcialmente determinado, pero a su vez el eje abierto-cerrado de una decisión-, esto no significa que la especificidad fenomenológica del significado esté adecuadamente reconocida por los enfoques de la teoría de sistemas. Y si es así, no podemos simplemente seguir al bioposthumanismo en su suposición de que nosotros, quienes estamos en un mundo significativo, somos animales humanos.

Para argumentar a favor de esta idea, debemos retornar a Heidegger y, específicamente, a su explicación de los animales como 'pobres-de-mundo'. Para hacer esto debemos examinar primero lo que Wolfe llama las "dos finitudes" que compartimos con "otros" animales: la finitud de la carne, encarnación, y "en última instancia, la muerte".

\section{La primera finitud: la vida como perturbamiento}

La explicación de Heidegger acerca del animal tiene algo similar a la idea de un código autopoiético. La "conducta" (Benehmen) de un animal es función de una autopoiesis particular a la especie -en el lenguaje de Heidegger, las "capacidades" que están establecidas por su "ser-impulsado por lo impulsivo" (1995, p. 237; 2007, p. 290)-, y tal "impulsar" permite cierta apertura, un "estar referido a..." o "un tender hacia" (1995, p. 240; 2007, p. 292). 
Esta apertura o acceso no debe ser entendida como si el mundo circundante (Unwelt) del animal fuera una totalidad de cosas presentes-a-la-mano, a las cuales éste se tuviera que adaptar; una totalidad que estaría igualmente disponible a otra especie de animal, en lugar de que el entorno perteneciera al impulso o al código mismo como su "apertura a través de la clausura" específica (Wolfe, 2010, p. xxi). Siguiendo a von Uexküll, Heidegger sostiene que el concepto "darwiniano" de adaptación defrauda en lo fundamental la "esencia de la animalidad" (Heidegger, 1995, pp. 260 y 277; Heidegger, 2007, pp. 314 y 335). En lugar de ello, el instinto comportamental del animal, que constituye su "apertura a...", lo mantiene dentro de una esfera propia de la especie o dentro de un "anillo" (Umring) -se está tentado a calificarlo como un cierto tipo de ser-en-el-mundo ${ }^{12}$. La vida del animal "es justamente el pugnar por este anillo que le rodea” (Heidegger, 2007, p. 309). Examinemos estas afirmaciones un poco más de cerca.

El término de Heidegger para la "totalidad del comportamiento instintivo" que pertenece al código autopoiético de una especie dada es "perturbamiento" (Benommenheit). El perturbamiento describe la manera específica del estar abierto del animal. En términos de Heidegger, el animal no "aprehende" 'lo que es', sino que es "tomado por las cosas" (1995, p. 242). La abeja, por ejemplo, no es atraída por el néctar de las flores como tal; más bien, su capacidad de impulso la provee instintivamente de un margen de maniobra (Spielraum) en el que ella puede ser "tomada" por el néctar. Las capacidades instintivas hacen esto (en el lenguaje de la teoría de sistemas) a través de la reducción de la complejidad, permitiendo al animal recibir información. En términos de Heidegger, el instinto del animal tiene un carácter "eliminativo" (1995, p. 250; 2007, p. 303): este excluye lo que no es relevante para la capacidad, permitiendo al animal, de este modo, ser estimulado por aquello a lo que está abierto. El "estímulo" no es un móvil libre del mundo circundante, sino un correlato del impulso animal; algo que desinhibe el carácter eliminativo del impulso en cuestión y, de esta manera, llama a un cierto modo de

\footnotetext{
${ }^{12}$ Aunque no debe ser llamado así. Cuando Heidegger dice cosas como "El animal tiene mundo. Del animal, pese a todo, no forma parte la carencia absoluta de mundo" (2007, p. 249), el contexto deja en claro que está proponiendo una interpretación (concretamente, el mundo consiste en "la accesibilidad de lo ente") (2007, p. 249) que cuestionará y rechazará en lo que resta del escrito. En contraste, su ponderado punto de vista es expresado en la afirmación de que el "perturbamiento es la condición de posibilidad de que el animal, conforme a su esencia, se conduzca en un medio circundante, pero jamás en un mundo" (2007, p. 291).
} 
la conducta del animal: la capacidad que define el funcionamiento de sus órganos (Heidegger, 1995, p. 253).

Con base en este análisis, entonces, se puede decir que cada especie animal consiste en el "anillo" constituido por la perturbación, en una conducta impulsiva-específica que dirige la especie. La abeja no ocupa el mismo entorno que la lombriz, el antílope o el homo sapiens. Ella es su mundo-circundante y puede comunicarse con otros mundos-circundantes únicamente al alcance que su estructura de impulso, o código, le permite precisamente para tales desinhibiciones. El animal, por consiguiente, no puede ser identificado en lo absoluto con el organismo en sentido estricto -esto es, con su cuerpo concebido como una entidad discreta entre otras entidades. El cuerpo del animal, por el contrario, pertenece $a$ su "anillo" como un órgano de su capacidad, pertenece al ser del animal como una "unidad del perturbamiento" (Heidegger, 1995, p. 258). Así, la idea del bioposthumanismo de que la "encarnación", la "finitud de la carne", tiene un solo significado a lo largo de la biósfera, que puede proveer una piedra angular para la teoría, parece simplista. Lo que nuestro "parentesco corporal con el animal" (Heidegger, 1993, p. 230; 2006, p. 31) augura, debe ser abordado de un modo diferente.

Heidegger se empeña en una larga explicación acerca del animal, con el fin de aclarar la tesis de que éste es pobre-de-mundo. Es sin embargo consciente de que su explicación puede sugerir la conclusión opuesta, porque en el reconocimiento del único tipo de ser del animal -concretamente, el anillo autopoiético y sus formas diversas de "apertura a..."-, percibe que lo denominado por él "pobreza de mundo" no debe ser entendido "simplemente" en un sentido negativo: "Los seres humanos quizás no tienen nada que ver" con los tipos de apertura disponibles de ser de una abeja o un delfín (Heidegger, 1995, p. 255). Aun así, Heidegger rehúsa declarar que cada una de estas especies ocupa un mundo, y acude a von Uexküll para criticar la conjetura de que el mundo humano pueda ser entendido en términos de la estructura del anillo de la animalidad (Heidegger, 1995, p. 263; 2007, p. 318). ¿Pero por qué no? Obviamente, si tomamos "mundo" para querer señalar la clase de círculo (ring) al que Heidegger da el nombre de "ser-en-el-mundo" en Ser y tiempo, podemos decir que los animales carecen de cierto aspecto de esto; pero por la misma razón, los seres humanos carecen del tipo de mundo constituido por la estructura instintiva de la abeja o del delfín. Heidegger mismo se percata de que podemos llamar a esta "diferencia" del animal, a su propia forma de "apertura perturbada", pobreza de mundo, únicamente "en comparación con 
el hombre" (1995, p. 270; 2007, p. 326), y se pregunta por qué deberíamos tratar esto así como si fuera la norma. ¿Es el hombre la medida de todas las cosas? Hallamos aquí el soporte de la decisión de Heidegger entre humanismo y posthumanismo.

¿Hay en la distinción entre Welt y Umwelt o contorno animal (Umring), algo más que una diferencia de los anillos entre sí? El curso de 1929/30 marca una transición en el preguntar, al sugerir que un análisis propio del mundo puede "obligarnos" en sí mismo a ver, después de todo, la "carencia" de mundo de los animales en términos de "privación o pobreza" (1995, p. 270; 2007, p. 326). Las partes del texto que posteriormente llevan a cabo este análisis, pueden ser interpretadas como el restablecimiento de un tipo de humanismo en el que una distinción dentro de la categoría general de vida, concuerda con una prioridad normativamente relevante para el ser humano. Pero esto no es, de hecho, la manera en que procede el argumento.

Esto es porque Heidegger da por sentado una de las premisas del bioposthumanismo, concretamente que el homo sapiens está transpuesto en el anillo circular de las cosas vivas (1995, p. 278; 2007, pp. 335-336). La ontología regional de la naturaleza o de la vida, que surge en la explicación heideggeriana del organismo, incluye a los seres humanos. La naturaleza no es "la suma total de entes presentes a la mano" ni es "el 'fundamento' sobre el que surge la "humanidad", como lo es en los humanismos teológico y tradicional (Heidegger, 1995, p. 278; 2007, pp. 335-336). La vida humana pertenece a la naturaleza. Como la abeja y el delfín, el homo sapiens tiene su propio ani110 -“(...) la naturaleza viviente nos mantiene apresados a nosotros mismos en tanto que hombres" (Heidegger, 2007, p. 336)- y, como ellos, estamos transpuestos en estos otros anillos de una manera tal que, estando disponible dentro de nuestro propio anillo de especie (Heidegger, 1995, p. 278; 2007, p. 336), se rechaza el modo de "acompañarlos". Aun así, Heidegger no se acoge al bioposthumanismo, sino que insiste en la importancia "metafísica" de una distinción entre mundo y anillo (ring). Si el mundo no es algo por lo cual "nosotros", los seres humanos, estamos "elevados" sobre la vida y la naturaleza, entonces ser-en-el-mundo no puede corresponder ontológicamente al ser humano. Tal y como Heidegger lo expresa en este curso, ser-enel-mundo es el "Dasein en nosotros", y el Dasein en nosotros no puede ser concebido como parte de nuestra estructura pulsional o código autopoiético. Nosotros, que somos en el mundo, nunca hemos sido animales. Hay una distinción ontológica que "trasciende" (Heidegger, 1995, p. 308; 2007, p. 369) 
el anillo-como apertura de las cosas vivas, así como también las categorías de vida. Pero por esta misma razón, esto no es algo que pueda soportar el tropo humanístico del "hombre" como medida.

\section{La segunda finitud: comportamiento, significado y lenguaje}

La discusión previa acerca del animal ha suprimido los pasajes en los cuales Heidegger anticipa la diferencia decisiva entre el anillo del animal y el mundo del Dasein. Como Heidegger enfatiza repetidamente, el animal tiene acceso a las cosas, pero no tiene acceso a ellas en tanto lo que ellas son (1995, p. $198 ; 2007$, p. 250). Esto significa que los animales no "aprehenden" las cosas, sino que son "tomados por" ellas. La forma de apertura que el perturbamiento del animal hace posible, su conducta, no le permite a la cosa "ponerse" o estar "presente" (Heidegger, 1995, pp. 252 y 242; 2007, pp. 293-294 y 305-306). En contraste, el comportamiento humano (Verhalten) -o, mejor, el comportamiento del Dasein- permite encontrar algo en tanto que algo. Para ponerlo esquemáticamente, el Dasein puede encontrar la roca en tanto que una roca (esto es, en el propio ser de la roca), mientras que el anillo de la lagartija tiene permitido acceder únicamente a la "roca-de-la-lagartija", el anillo del escarabajo tiene permitido acceder únicamente al "camino-del-escarabajo" (Heidegger, 1995, p. 198; 2007, p. 249) ${ }^{13}$. La pregunta obvia es: ¿por qué esto no es sencillamente lo mismo en el ser humano, quien debería tener acceso únicamente a la "roca-del-humano" o al "camino-del-humano"?

Todo gira aquí en torno a entender que el camino de una fenomenología del significado requiere una distinción que no puede ser suscitada dentro del horizonte conceptual de una metafísica de la vida. Al mismo tiempo debemos recordar un punto anotado anteriormente: la fenomenología heideggeriana no está orientada a proveer algo, sino a llevarnos a una situación en la cual

\footnotetext{
${ }^{13}$ McNeill (1999, p. 240) especula que los animales deben ser capaces de encontrarse con las cosas en tanto que "algo" (por ejemplo, como comida), pero no en tanto que "entes". Yo no pienso que este sea el punto de vista de Heidegger, sino que parte del asunto debe ser meramente terminológico. Si el "en tanto que" significa que el animal habita lo que la teoría de sistemas llama la "virtualidad" del significado, entonces, ya que esto comprende una capacidad de respuesta a la posibilidad en tanto que posibilidad, y puesto que esto no parece ser parte de la capacidad del animal, en este sentido el animal no puede encontrar las cosas "en tanto que". Pero si esto únicamente significa que el ser "tomado" del animal por las cosas comprende la expresión de capacidades diferenciadas, entonces, ciertamente los animales están abiertos a las cosas en tanto comida, en tanto refugio, y así sucesivamente.
} 
sea posible decidir (krinein) cuáles son los riesgos de la pregunta (Heidegger, 1995, p. 351).

Nos limitaremos a tres tesis que rigen la reflexión de Heidegger: 1) la estructura del "en cuanto" ( $A l s)$, o significado, no está fundamentada en el lenguaje (logos), sino que fundamenta al lenguaje; 2) el "en cuanto", expresado en la cópula ( es b), indica formalmente la "dimensión" en la que esta estructura debe ser entendida fenomenológicamente; concretamente, "el Dasein en nosotros" y, 3) esta dimensión no es un sistema autopoiético o un anillo animal, sino un tipo de "comportamiento fundamental" que hace posible la manifestación de los entes como tal, junto con un "temple de ánimo fundamental" que hace posible la manifestación de los entes como un todo (diferencia ontológica). Estas aseveraciones circunscriben conjuntamente lo que significa sostener que el "hombre" es "configurador-de-mundo" (Heidegger, 1995, p. 285; 2007, p. 344), e indican por qué razón, como Heidegger lo expresa, la "consciencia" (o el sistema intra-psíquico autopoiético del animal) "no es suficiente" para configurar mundo (Heidegger, 1995, p. 361; 2007, p. 427).

Empecemos con el ejemplo de Heidegger acerca de la estructura del "en cuanto". Cuando estoy impartiendo una lección ante una clase, puedo hacer la vivencia del tablero como (als) estando en una mala posición. En una vivencia como esta no tengo simplemente acceso a los entes; más bien, éstos se manifiestan en cuanto algo -como un tablero y en mala posición. Si yo expreso ahora esto como "el tablero está en una mala posición", el enunciado no establece en primer lugar el "en cuanto", sino que lo presupone: "La proposición «a es b» no sería posible (...) si ella no pudiera surgir de la vivencia que subyace al a en cuanto b" (Heidegger, 1995, p. 301). El último es un tipo de "percibir que configura unidad" (Heidegger, 1995, p. 314; 2007, p. 375), y que precediendo permite el rasgo característico del enunciado: su virtud de ser verdadero $o$ falso. Puesto que Heidegger, en el curso en cuestión, rastrea el origen de estos asuntos hasta Aristóteles, puede decir que "el logos se fundamenta, conforme a su posibilidad interna, en el nous" (Heidegger, 1995, p. 314; 2007, p. 375).

Como Luhmann, el Aristóteles de Heidegger concibe el lenguaje (logos) ante todo como comunicación: "El discurso da a entender y exige comprensión. Conforme a su esencia, se dirige al libre comportamiento y actuar de los hombres entre sí" (Heidegger, 1995, p. 306; 2007, p. 367). Pero para Heidegger, en contraste con Luhmann (quien sigue la tradición que Heidegger está 
criticando), la comunicación es secundaria en relación con la "aprehensión original que forma una unidad" (nous); es decir, no es un sistema social autopoiético. Tanto la teoría de sistemas como aquello que Heidegger denomina la tradición "lógica" -que deriva del análisis aristotélico de la enunciaciónanalizan el "en cuanto" mismo como una función del "es" de la enunciación, de la cópula. De este modo, ellos reducen la unidad producida a una relación formal entre términos y, en últimas, a "un tecnicismo o mecanicismo radicalmente a-humano" (Wolfe, 2010, p. 88). Al hacer esto, la lógica entiende el carácter distintivo del ser humano como algo que reside en la razón en tanto capacidad de operar con relaciones formales. Pero Heidegger ofrece una explicación alternativa en la cual el "en tanto que" se convierte en el "es" de la predicación únicamente en la comunicación, y la razón (o nous) precede a la lógica.

Esto nos lleva a nuestra segunda tesis, que concierne a la dimensión fenomenológica formalmente indicada por la cópula (Heidegger, 1995, p. 291). Si bien es verdad que "a en cuanto b" es formalmente una relación, la noción de relación es filosóficamente inútil porque postula automáticamente "a" y "b" como entidades "presentes". La fenomenología, en contraste, tomará esta relación formal entre a y b como una indicación o guía hacia "la tarea decisiva de concebir la relación a partir de su dimensión propia” (Heidegger, 1995, p. 293; 2007, pp. 352-353). ¿Cuál es entonces la dimensión propia del "en cuanto" o del significado? En sus primeros cursos de Friburgo, lo que Heidegger denomina "dimensión propia" era expresado como "Evidenz-Situation", la experiencia concreta en primera persona, en la que el tipo específico de relación en cuestión puede ser "intuido en persona", en su ser genuino ${ }^{14}$. Para tener éxito en esta tarea formalmente indicada-ocupar la dimensión apropiada o la situación de evidencia- hay que pensar filosóficamente. Pensar, en este sentido fenomenológico, es aceptar el tipo de aprehensión que pertenece al nous. En contraste con la tradición lógica, que no presta atención a la dimensión propia y trata la cópula como formalmente vacía, la fenomenología heideggeriano-aristotélica descubre en la cópula una articulación interna en el ser-qué (what-being), ser-que (that-being) y ser "en el sentido de verdadero" (Heidegger, 1995, p. 326; 2007, p. 389); una articulación cuya unidad deriva de la dimensión del "en cuanto". De esta manera, si el "logos se funda,

${ }^{14}$ Para una explicación de la indicación formal en términos de Evidenz-Situation, obsérvese Crowell (2001, cap. 7). 
conforme a su posibilidad, en el nous" -es decir, en "un percibir que configura unidad" (Heidegger, 1995, p. 314; 2007, p. 375)-, el pensar que pretenda ocupar la dimensión a la cual pertenece el significado (el "en cuanto") será un pensar en sí mismo, será un "tomar en consideración"15 o un aceptar el camino en el que acontece el significado.

Los detalles pueden ser aquí controversiales, incluso misteriosos. Nuestra preocupación no es, sin embargo, en torno a ellos, sino acerca del posthumanismo de Heidegger, acerca de la comprensión del "Dasein en nosotros" que surge de la discusión. En este sentido bien podría parecer que Heidegger está simplemente repitiendo los rasgos más comunes del humanismo, en los cuales el significado y el lenguaje están fundados en el nous (reason) que, aceptando la forma en que acontece el significado (thinking), tiene la capacidad -negada a los sistemas autopoiéticos- de comprender las condiciones de posibilidad de su propio funcionamiento. Pero mientras las relaciones conceptuales son aquí abrumadoras, la fenomenología es relativamente sencilla y puede ser abordada observando que Heidegger identifica el nous con el comportamiento. Como "aprehensión original que forma una unidad", el nous pertenece a todo comportamiento (Heidegger, 1995, p. 342; 2007, p. 406), y es a través de la fenomenología de este último -que en Ser y tiempo se llama el "por-mor-de-qué" (Worumwillen) - que podemos entender la naturaleza del nous como la dimensión del significado.

En contraste con la conducta vital del animal, el comportamiento es una capacidad existentiva que involucra al ser en aras de algo. Pero retornemos a nuestro ejemplo: puedo tener la vivencia del tablero como estando en una mala posición porque no puedo hacer con él lo que debo llevar a cabo (concretamente, escribir en él de una manera tal que los estudiantes puedan verlo). Pero yo puedo experimentar la insuficiencia de mi habilidad de usarlo adecuadamente, solo porque estoy comprometido en cierto tipo de comportamiento - esto es, porque hago lo que hago en aras de ser un profesor. Esta no es una habilidad o capacidad, sino una "posibilidad" existentiva; algo cuyo ejercicio requiere que yo sea consciente de mí mismo en cuanto que posiblemente tenga éxito o fracase. De este modo el comportamiento es una especie de norma-regulada tratando de ser. Al ejercitar mi capacidad para ser

${ }^{15}$ Traducción de Heidegger de noein en ¿Qué significa pensar? (1968, p. 207; 2005, p. 170). La versión alemana (Heidegger, 1971, p. 172) dice: "nous bedeutet das Sinnen, das etwas im Sinn hat und sich zu Herzen nimmt". Acerca de todo esto, cfr. Crowell (2016). 
un profesor, estoy tratando de cumplir con los estándares que configuran lo que es o significa ser un profesor. Si no estuviera normativamente orientado de esta manera, el tablero podría no presentarse a sí mismo como en una mala posición. De acuerdo con Heidegger, ningún animal trata de ser una abeja, un delfín, o un "humano", aunque todos ellos puedan tener éxito o fracasar al hacer lo que su estructura instintiva les provee con una capacidad para hacerlo. Contrariamente, el comportamiento está esencialmente orientado hacia una medida de éxito o fracaso, que aunque indefinida puede ser algo que yo puedo tratar de satisfacer. Solamente porque el comportamiento implica este aspecto normativo, los entes pueden estar ahí para mí en cuanto tales -esto es, en tanto entes, en tanto lo que ellos son y en tanto eso que son en realidad. El comportamiento es el origen de la "multiplicidad indivisa" expresada en la cópula (Heidegger, 1995, p. 333; 2007, p. 396).

La idea de que el comportamiento (nous) fundamenta el significado (el en cuanto), es central en la distinción heideggeriana entre el homo sapiens y el "Dasein en nosotros", y así también en la diferencia entre bioposthumanismo y posthumanismo fenomenológico. Desde el punto de vista fenomenológico, el tipo de expresión que puede ser verdadero $o$ falso depende del comportamiento. Como una realización que incorpora una norma, es posible únicamente dentro de la dimensión de algo que nosotros estamos tratando de ser. Por consiguiente, esta habilidad no es una "capacidad", y no puede ser concebida como una función del código autopoiético del animal humano (Heidegger, 1995, p. 337; 2007, pp. 400-401). Heidegger caracteriza esta distinción con el término "libertad". El comportamiento es "ser libre para los entes en cuanto tales", precisamente porque su propio ser es normativamente la cuestión que él trata de llevar a cabo.

El bioposthumanismo denomina este "estar-en-cuestión" la "virtualidad" del significado: el hecho de que todo objeto deseado implique un horizonte de posibilidad y, de esta manera, la "presentación simultánea de actualidad y posibilidad" (Wolfe, 2010, p. 16). Pero esto traza un código autopoiético a este fenómeno, cuyas normas (si hay normas) no pueden convertirse en un conflicto al interior del sistema de significado. De acuerdo con Heidegger, sin embargo, si las medidas son esenciales para el tipo de posibilidades del horizonte que acompañan la presentación de un objeto, y no pueden estar explícitamente en riesgo por el sistema en sí mismo, éste no puede ser entonces un sistema de significado en lo absoluto. El tipo de posibilidad requerido para la experiencia de algo en tanto que algo demanda libertad, un 
“(...) libre ofrecerse, dejándose vincular, a aquello que está dado como ente" (Heidegger, 1995, p. 342; 2007, p. 406). Tal libertad no es una facultad misteriosa que nos saca del orden causal; es un "estar abierto a los entes" por medio de la necesidad de medidas normativas en tanto que normativas. En sentido heideggeriano, la libertad está siempre demarcada, pero tal demarcación es totalmente diferente al perturbamiento autopoiético del animal. El "mundo" es la diferencia entre una aprehensión normativamente demarcada de entes en cuanto tales y una "apertura a" perturbada.

La fuerza de este argumento puede ser observada si regresamos al análisis realizado por Heidegger acerca del enunciado. El enunciado "a es b" es un enunciado únicamente si, afirmándolo, estoy orientado hacia la posibilidad de la verdad $o$ la falsedad -esto es, orientado hacia una distinción normativa en la cual las cosas en el mundo son reconocidas como medidas de lo dicho por mí. Pero ¿cómo es posible esto? ¿Cómo se convierten las cosas en medidas? Un sistema autopoiético puede tener "interferencia" - 0 , en términos de Heidegger la 'vida', en tanto que perturbamiento, puede ser tomada por las cosas de modo que éstas estimulen sus capacidades-, pero carece de recursos para virtualizar su entorno a través de la medida. De este modo carece de una condición para el significado (mundo). El comportamiento del Dasein provee la condición que hacía falta. El comportamiento particular que hace una enunciación (un "señalar que puede ser verdadero $o$ falso"), por ejemplo, implica una "(...) adecuación a (...) y una vinculación a (...), de tal modo que aquello con lo que se establece la vinculación, lo ente, se manifiesta en su vinculatoriedad" (Heidegger, 1995, p. 339; 2007, p. 403). De esta manera el comportamiento lleva a cabo el espacio de juego (Spielraum) que abre lugar a una verdad $o$ falsedad regida normativamente.

Esto puede ser ilustrado regresando a nuestro ejemplo, para con ello mostrar cómo el comportamiento suscita la articulación interna de la cópula. Ya hemos visto que el tablero puede presentarse estando en una mala posición únicamente porque yo estoy tratando de hacer uso de él con el objetivo de ser un profesor. Tratar de ser un profesor requiere que yo sea libre, es decir, que sea capaz de "dejarme vincular" por las medidas de la enseñanza (Heidegger, 1995 , p. 342; 2007, p. 406). Pero al dejarme a mí mismo ser regido (ser vinculado) de esta manera, este "dar la medida" necesariamente "(...) se transfiere de entrada, en el sentido del comportamiento que se deja vincular, a lo ente, de modo que en éste se regula la adecuación y la inadecuación" (Heidegger, 1995 , p. 342; 2007, p. 406). Por consiguiente, el tablero puede mostrarse 
como estando en mala posición porque los entes que hacen parte del ámbito del enseñar tienen una virtualidad $u$ horizonte de actualidad-posibilidad muy específica y normativamente regida. Los tableros, se supone, existen para estar de esta o aquella manera; su "ser-qué" les provee la medida. Este ("serqué") no cumple la medida a causa de algún defecto propio, sino únicamente porque yo mismo me he dejado vincular por las medidas de la enseñanza. Finalmente, mi enunciado de que el tablero está en mala posición establece, como su medida, el ser de verdad de la cosa en mala posición: la cosa misma ("ser en el sentido de verdad") mide mi comportamiento debido a que en la enunciación actúo en aras de señalar lo que en verdad es la cosa; esto es parte de lo que está en juego en aquello que estoy tratando de ser. Puesto que todo comportamiento está vinculado con una distinción normativa entre éxito y fracaso, Heidegger puede denominarlo "(...) manifestabilidad antipredicativa, o mejor, verdad prelógica" de las cosas (1995, p. 341; 2007, p. 405).

La explicación que Heidegger hace del significado se convierte así en algo que elude totalmente el concepto de sistema psíquico de la teoría de sistemas, concretamente, la orientación hacia la dimensión de la primera persona. La posibilidad intrínseca de la estructura del "en tanto que" (Heidegger, 1995, p. 335; 2007, pp. 338-339) depende del comportamiento. El comportamiento implica el hecho de ser en aras de algo, en el que el ser humano se mantiene junto con otras cosas que le permiten convenir (come into agreement) con ellas (Heidegger, 1995, p. 308; 2007, pp. 368-369), para aprehenderlas en lo que ellas son. Este 'convenir' no está, sin embargo, simplemente dado: al mantenerme en mi comportamiento junto con las cosas, puedo fracasar al (buscar) aprehenderlas en lo que son. Todo esto explica por qué Heidegger puede sostener que "el «ser» y el «en tanto que» apuntan al mismo origen" (1995, p. 333; 2007, p. 397): la necesidad normativa del comportamiento es la raíz común de ambos (2007, p. 402), y ésta constituye la diferencia ontológicamente decisiva entre el anillo del animal y el mundo.

De esta manera (en 1929/1930), Heidegger no encuentra en la posesión del lenguaje $^{16}$ la diferencia entre seres humanos y animales. Más bien sitúa a

\footnotetext{
${ }^{16}$ McNeill (1999, p. 234) reconoce este "arraigo del logos" -y esto incluye "cada logos"- "en el acontecer del mundo". Aunque también sostiene que el "traer a la presencia" que el Dasein en nosotros cumple -algo que el animal es incapaz de hacer- es una función del lenguaje: "Al ser traídas al lenguaje, las cosas también logran, en primera instancia, una cierta resistencia y permanencia de la presencia; pueden estar frente a nosotros (...) en varios tipos de presencia a la mano" (1999, p. 239). Pero mientras que la presencia-a-la-mano puede requerir del lenguaje, esto no es "lo que nosotros denominamos ser". Puesto que una condición del lenguaje en sí mismo es que el Dasein
} 
ésta como previa al lenguaje, en una especie de aprehensión, regulada por normas, que forma una unidad. El significado, el rasgo fundamental del mundo, es exclusivamente este tipo de aprehensión. En contraste con la estructura "indicativa" de la comunicación del animal, el comportamiento lingüístico no deriva de un sistema autopoiético psíquico o social, sino del "Dasein en nosotros". Y puesto que el Dasein en nosotros nunca ha sido animal, no está expuesto a la segunda finitud que el bioposthumanismo asegura que es ignorada por la tradición fenomenológica: el sometimiento del significado al juego de la iteración y del signo, del "tecnicismo y el mecanicismo a-humano" de los sistemas de la semiótica ${ }^{17}$. Por supuesto, la comunicación del Dasein, su empleo de signos, no puede evitar las coacciones de la semiosis: Sorge no sirve para "fijar el referente" más allá de todo juego de significantes. Pero esto no debilita la tesis fenomenológica de que el cuidado, la orientación hacia la medida en un ser cuyo propio ser es un problema para él, es el fundamento de una diferencia ontológica entre el entorno del animal y el mundo.

Pero si la orientación hacia una norma destaca en todos sus comportamientos - es decir, si la libertad de (tratar de) ser algo pertenece al Dasein en cuanto tal-, ella no relata aún la historia completa acerca de cómo el "es" y el "en tanto que" surgen con tal comportamiento, ya que el origen de la unidad indiferenciada que se expresa en la cópula (el significado del "ser") no puede ser rastreado en ningún comportamiento específico, como cuando se trata de ser un padre o un filósofo ${ }^{18}$. La manifestación de los entes "en cuanto tal" y "en tanto que un todo" (mundo), está cimentada en un comportamiento fundamental y en un temple de ánimo fundamental.

debe ser capaz de tomar una posición, orientarse a sí mismo hacia un tipo de medida a la cual se vincule -por lo menos para el Heidegger de la "década metafísica". McNeill parece introducir en sus análisis la concepción posterior del lenguaje en Heidegger, aunque esta concepción no tiene nada que ver con el tipo de crítica semiótica que se constituye en el punto de discusión entre Heidegger y los bioposthumanistas.

${ }^{17}$ Esta tampoco es la posición expuesta por Heidegger en la crítica derridiana, según la cual la fenomenología está incapacitada por la operación de "iteración" que pertenece a todos los sistemas de signos. En contraste con la imagen fonocéntrica criticada por Derrida, Heidegger concibe que no es el caso en el que "el hombre tiene emisión de voz que, en adición, tiene un significado conectado a ésta" -digamos, por alguna intención interna de la consciencia (Heidegger, 2007, p. 367). Más bien, es porque nuestra esencia aprehende [el nous, es decir, la libertad en el comportamiento] el significado que tienen nuestras emisiones.

${ }^{18}$ En un interesante pasaje, Heidegger hace referencia a lo que parece ser una implicación de su análisis; concretamente, que no hubo Dasein (entendimiento del ser) antes de que los seres humanos comenzaran a filosofar. Rechaza sin embargo esto (2007, pp. 420-421).

Crowell, S. (2020). Nunca hemos sido animales. El posthumanismo de Heidegger. 


\section{La diferencia ontológica ${ }^{19}$}

En Ser y tiempo Heidegger había analizado el "comportamiento fundamental" como muerte existencial, la capacidad-de-ser radicalmente en primera persona "sí-mismo" del Dasein, incluso cuando su habilidad-para-ser-algo (profesor, padre) fracasa. En el curso de 1929/30 Heidegger llama a esta capacidad ser 'sí-mismo': "el ser libre en un sentido original" (Heidegger, 1995 , p. $343 ; 2007$, p. 407). La libertad originaria no es un comportamiento guiado por las medidas de alguna identidad específica, sino por la responsabilidad de medir en cuanto tal. Ser responsable por ser es lo que está ahí por ser Dasein; éste provee "la medida para toda medición" (Heidegger, 1995, p. 346; 2007, p. 410). Por esta razón él es la base sobre la cual puede aparecer 'lo que es' en tanto ente (ente en cuanto tal). Para ocupar esta dimensión del "en tanto que", esta dimensión de responsabilidad -el comportamiento fundamental de la libertad originaria-, se debe asumir "(...) sobre nosotros el esfuerzo de transformar al hombre (...) en una existencia más original": el Dasein en nosotros (Heidegger, 1995, p. 350; 2007, p. 415). De esta manera, este posthumanismo fenomenológico no concibe al ser humano ni al Dasein como la medida; más bien, el Dasein está obligado a la medida, que precisamente en tanto que medida, trasciende su "poder"20.

Esto se evidencia también en el "temple de ánimo fundamental", que junto con el comportamiento fundamental forma parte esencial de la responsabilidad. En la disposición afectiva de la angustia (o en el "aburrimiento" fundamental de 1929/30), los entes en tanto totalidad se hacen fenomenológicamente patentes en su peculiar "integridad"21. Esto no significa que la totalidad de los entes esté presente para mí a la mano. Más bien significa que

\footnotetext{
${ }^{19}$ Para una discusión detallada de los análisis mencionados aquí, cfr. Crowell (2013), especialmente la parte II.

${ }^{20}$ Obsérvese la discusión en Ser y tiempo: "El sí-mismo que, como tal, tiene que poner el fundamento de sí mismo, jamás puede adueñarse de éste" (Heidegger, 1962, p. 330; 1997, p. 279). La medida tampoco puede ser equiparada con el Ser (Sein) en sí mismo, puesto que "el «ser»y el «en tanto que» apuntan al mismo origen", es decir, el comportamiento. La medida hacia la cual el comportamiento está orientado y que trasciende su poder está, así, "más allá del ser".

${ }^{21}$ Tal como en todo comportamiento, los entes "en cuanto tal" son de alguna manera el asunto más importante. De esta manera, en todos los temples de ánimo, los entes "en tanto que un todo" importan de algún modo. Pero esta estructura se hace evidente gracias al "sistema" (Dasein), cuya estructura está solamente en ciertos comportamientos y modos distintivos que son, por consiguiente, metodológicamente privilegiados. Acerca de la distinción metodológica del Angst, cfr. Heidegger (1962, pp. 228ss.; 1997, p. 206).
} 
lo que es, en tanto un todo, importa de alguna manera para mí, "previo a toda toma de postura" (Heidegger, 1995, p. 353; 2007, p. 419). En contraste con el carácter selectivo de la estructura de impulso del animal, el temple de ánimo fundamental es un tipo de "cumplimiento", el "predominante" del mundo (Heidegger, 1995, p. 351; 2007, p. 416).

Heidegger llega a este punto haciendo referencia a una objeción de la que ha sido objeto por parte de generaciones de filósofos. Si yo rastreo el origen de la mala posición del tablero en relación con mi comportamiento (por ejemplo, mi intento de ser un profesor), ¿no es esto constituirme en el sentido de que su mala posición es relativa a mi propia posición? ¿No es "subjetivizar el significado al que el ente en cuestión está direccionado? Heidegger responde que el tablero está en mala posición no solamente en relación a mi posición. Él está "en sí mismo" en mala posición en relación con "toda" el aula de clases (1995, p. 344; 2007, p. 408). Mi intento de ser algo, en sí mismo, no configura el mundo; lo que lo configura es mi intento, junto con la manera en que las cosas -en tanto que un todo- "prevalecen" o hacen que se logre esto de forma efectiva. Otorgando importancia al develamiento de una esfera en la cual estoy "arrojado" -pero dado que tal arrojamiento es inseparable del comportamiento-, mi esfera o mundo no puede ser visto como una versión del perturbamiento del anillo del animal ${ }^{22}$. En lugar de ello el temple de ánimo fundamental implica que las medidas a las cuales se ata el comportamiento, y por las cuales yo-mismo soy responsable, no son meros datos dados

\footnotetext{
${ }^{22}$ Por esta razón no puedo estar de acuerdo con la propuesta de McNeill de que nuestro temple de ánimo atestigua nuestra propia forma de "perturbamiento" (animal). Después de citar un pasaje en el que Heidegger declara que "el temple de ánimo justamente nos hace manifiesto lo ente en su totalidad", no como un objeto sino como una forma de "ser traído a la presencia (...) y eso significa a la manifestación de la existencia en cuanto tal, según se halla en cada caso en medio de esta totalidad". McNeill sostiene: "la primacía del temple de ánimo en la revelación de nuestro propio ser conlleva a que la naturaleza viviente, al mantenernos apresados [mi énfasis], nunca sea reducible a un objeto de contemplación teórica. En lugar de esto, es decir a lo que siempre estamos atados con antelación; que nos ata [mi énfasis] previo a todas nuestras actividades y acciones" (1999, p. 236). Pero en ninguna parte del pasaje citado (y en ninguna otra parte que yo conozca) Heidegger dice que la naturaleza ("nuestro ser", el Dasein en cuanto tal) nos mantenga apresados. El tipo de atadura de los entes en tanto que un todo que pertenece al temple de ánimo, no menos que el tipo de atadura como tal que pertenece al comportamiento debe ser pensado en términos de la diferencia ontológica entre el homo sapiens y el Dasein. No podemos tratar lo que el temple de ánimo revela simplemente como un tipo de hecho dado; la marca de nuestra "pertenencia" a "la naturaleza viva", ni podemos contrastar este aspecto de nuestro ser con otro aspecto "histórico". El Dasein no es "el animal histórico" más de lo que es "el animal racional".
} 
(das Man) ni simples medidas mías para inventar. Ellas se ponen en juego en aquello que estoy haciendo dentro del todo que impera.

La unidad original del comportamiento y del temple de ánimo fundamental -el cimiento para la diferencia ontológica entre ser y entes en cuanto tales, y en tanto que un todo- explica por qué el Dasein no está sujeto a la objeción de que un sistema autopoiético no pueda operar de acuerdo con su código autorreflexivo y simultáneamente identificar la lógica de esta operación del código. Puesto que el Dasein en nosotros no es vida o animalidad, opera de acuerdo con normas, pero no a la luz de ellas. Más bien es el margen de acción en el cual todo código o medida es puesto en discusión. El "código" del Dasein es un problema por sí mismo si se insiste en ponerlo de esa manera. De esta forma, ni la primera finitud de la encarnación (muerte, sufrimiento) ni la segunda finitud de la diferencia semiótica pueden llegar al Dasein en nosotros. Nosotros, Dasein, nunca hemos sido animales.

¿Qué distinción genera esta "diferencia ontológica" en relación a las preguntas (sobre todo a las éticas) que motivan el bioposthumanismo? ¿En qué sentido esta diferencia es normativamente más importante (constituyendo un abismo) que todas las otras diferencias que separan al homo sapiens y a las otras especies de animales? En respuesta a estas preguntas debe decirse que esta diferencia es solo más importante dados los intereses de la filosofía (o "teoría"). Si lo que tenemos que hacer es encontrar nuestro camino en la oscuridad, entonces la capacidad del murciélago, debido a su radar de navegación, bien puede ser más importante que la diferencia ontológica. Pero si lo que está en riesgo es lo que nosotros debemos entender por ética -por nuestra responsabilidad con nuestro comportamiento hacia los otros (incluyendo otros animales)-, entonces estamos confrontados con una pregunta de riesgos y medidas como tal. Si Heidegger está en lo correcto, tal preocupación está presente solo en los entes con la estructura del Dasein; entes que pueden asumir responsabilidad en relación con la medida, porque en su comportamiento aquello que significa 'ser' es (normativamente) el problema a discutir.

Afirmar con el bioposthumanismo (siguiendo a Bentham), que la pregunta ética no es "¿pueden ellos hablar o pueden ellos razonar, pero no pueden sufrir?" (Wolfe, 2010, p. 81), es tomar una posición, decidir (krinein) lo que la medida de nuestro ser debe ser. Esto ya opera, por tanto, dentro de la diferencia ontológica, y se erige a sí mismo como un modelo acerca de la mejor 
manera de continuar nuestro trato con los animales. Pero la preocupación que motiva al bioposthumanismo no puede de ninguna manera surgir desde el sistema que teoriza y deconstruye. Tomar el "sufrimiento" como una consideración normativamente relevante (y de hecho predominante) solamente es posible por parte de nosotros, que nunca hemos sido animales. Nosotros -el Dasein en nosotros- no podemos escapar de la responsabilidad a través del recurso a una teoría que prescinda de la filosofía en general, con el fin de plantear preguntas críticas a "nosotros" en nombre de "otro animal". El post-humanismo de Heidegger viene tanto antes como después del animal humano, y a pesar de su afirmación al final del curso impartido entre 1929 y 1930, que sostenía que "También la filosofía trascendental tiene que caer" (1995, p. 359; 2007, p. 425), la fenomenología trascendental es la única manera de dar sentido a este tipo de asuntos.

\section{Referencias}

Barbaras, R. (2006). Desire and Distance: Introduction to a Phenomenology of Perception. (Trad. Milan, P). Stanford: Stanford University Press.

Crowell, S. (2001). Husserl, Heidegger, and the Space of Meaning: Paths Toward Transcendental Phenomenology. Evanston: Northwestern University Press.

Crowell, S. (2013). Normativity and Phenomenology in Husserl and Heidegger. Cambridge: Cambridge University Press.

Crowell, S. (2014). "Transcendental Life." Phenomenology and the Transcendental. Heinämaa, S., Hartimo M., and Miettinen, T (Eds.), Phenomenology and the Transdendental (pp. 21-48). London: Routledge.

Crowell, S. (2016). What Is It to Think?. Breyer, Th. and Gutland, Ch. (Eds.), Phenomenology of Thinking (pp. 183-206). London: Routledge.

Derrida, J. (1994). Márgenes de la filosofía (Trad. C. González Marín). Madrid: Cátedra.

Figal, G. (2010). Objectivity. The Hermeneutical and Philosophy. (Trad. George, Th). Albany: SUNY Press. 
Foucault, M. (2010). Las palabras y las cosas: una arqueología de las ciencias humanas. (Trad. Frost, E. C). México: Siglo XXI.

Heidegger, M. (1962). Being and Time. (Trad. Macquarrie, J. and Robinson, E). New York: Harper \& Row.

Heidegger, M. (1968). What Is Called Thinking?. (Trad. Gray, J. G). New York: Harper \& Row.

Heidegger, M. (1971). Was Heißt Denken? Tübingen: Max Niemeyer Verlag. Heidegger, M. (1972). On Time and Being. (Trad. Stambaugh, J). New York: Harper \& Row.

Heidegger, M. (1993). Letter on Humanism. Basic Writings. (Trad. Krell, D. F). (pp. 193-242). New York: Harper Collins.

Heidegger, M. (1995). The Fundamental Concepts of Metaphysics: World, Finitude, Solitude. (Trad. McNeill, W. and Walker, N). Bloomington: Indiana University Press.

Heidegger, M. (1997). Ser y tiempo. (Trad. Rivera, J. E). Santiago de Chile: Editorial Universitaria.

Heidegger, M. (2000). Tiempo y ser. (Trad. Garrido, M., Molinuevo, J. L. y Duque, F). Madrid: Tecnos.

Heidegger, M. (2005). ¿Qué significa pensar? (Trad. Gabas, R). Madrid: Trotta.

Heidegger, M. (2006). Carta sobre el Humanismo. (Trad. Cortés, H. y Leyte, A). Madrid: Alianza.

Heidegger, M. (2007). Los conceptos fundamentales de la metafisica: mundo, finitud, soledad. (Trad. Ciria, A). Madrid: Alianza.

Lawlor, L. (2006). The Implications of Immanence: Toward a New Concept of Life. New York: Fordham University Press.

Maturana, H. y Varela, F. (1996). El árbol del conocimiento: las bases biológicas del conocimiento humano. Santiago de Chile: Editorial Universitaria. 
McNeill, W. (1999). Life Beyond the Organism: Animal Being in Heidegger's Freiburg Lectures 1929-30. Animal Others: On Ethics, Ontology, and Animal Life (pp. 197-248). Albany: State University of New York Press.

Toadvine, T. (2009). Merleau-Ponty's Philosophy of Nature. Evanston: Northwestern University Press.

Weigelt, C. (2002). The Logic of Life: Heidegger's Retrieval of Aristotle's Concept of Logos. Stockholm: Almqvist \& Wiksell International.

Wolfe, C. (2010). What is Posthumanism? Minneapolis: University of Minnesota Press. 